\title{
In Silico Analysis of Antimicrobial Peptides from Plants
}

\author{
Marian BUTU ${ }^{1)}$, Steliana RODINO*1,2), Petruta PETRACHE ${ }^{2)}$, Alina BUTU ${ }^{1)}$ \\ ${ }^{1)}$ National Institute of Research and Development for Biological Sciences, Splaiul Independentei 296, \\ 060031Bucharest, Romania \\ 2)University of Agronomic Science and Veterinary Medicine, 59 Marasti Bv., 011464 Bucharest, \\ Romania; corresponding author email: \\ *Corresponding author, email: steliana.rodino@yahoo.com
}

Bulletin UASVM Animal Science and Biotechnologies 71(2) / 2014,

Print ISSN 1843-5262; Electronic ISSN 1843-536X

DOI:10.15835/buasvmcn-asb:10532

\begin{abstract}
Plants are constantly exposed to different and varied stress factors. In these conditions plants synthesize antimicrobial peptides as innate host defense response. The investigation of the properties of the antimicrobial peptides is a field of remarkable interest to discover its mechanism of action and develop strategies for new biotechnological applications in medicine and plant protection. In this work were studied the thermodynamic parameters of interactions and the structure stability of defensin Lc-def from germinated lentil (Lens Culinaris) seeds by molecular dynamics simulation.
\end{abstract}

Keywords: antimicrobial peptides, plant defensins, molecular dynamics, simulations

\section{INTRODUCTION}

Antimicrobial peptides have important roles in the innate immune defense. Plants synthesize antimicrobial peptides as response of the innate defense system to physical, chemical or biological stress factors (Butu and Butu, 2011a). Plant defensins are antimicrobial peptides with 45-55 amino acid residues and have eight conserved Cys residues that build four disulfide bridges (de Oliveira Carvalho and Moreira Gomes, 2009). Plant defensins display a range of activities including antifungal and antibacterial activities, enzyme inhibitory activities as well as roles in heavy metal tolerance and development (van der Weerden and Anderson, 2014).

The computational methods have become a tool widely used in the last 10 years to study the structure of the antimicrobial peptides (Butu and Butu, 2011a). Computational methods can be considered as a bridge between theories and experiment and allows: extracting the results from a particular model; comparing experimental results; comparing theoretical prediction of a system with experimental behaviour; better understanding and interpretation of experimental observations; details correlation at the atomic and molecular level with macroscopic properties of system; providing the information which cannot be obtained from real experiments. Knowledge of the structure of the antimicrobial peptides from plants (AMPs-P) and their mechanisms of action is particularly important for the plant protection domain. Also, AMPs-P are an valuable source of natural antibiotics for human and veterinary medicine (Butu and Butu, 2010; 2011a).

The important progresses in the computing resources have given the possibility of comparison of simulation data with experimental data, of refinement of experimental data, of replacing laboratory experiments with simulation predictions, all at once, saving time and financial resources (Butu and Butu, 2011a). In this research, we applied bioinformatics methods to study the potential biological activity of AMPs-P. 


\section{MATERIALS AND METHODS}

The antimicrobial peptide used in the simulation experiments was defensin Lc-def from germinated lentil (Lens Culinaris). Its structure was taken from the scientific database RCSB PDB (structure ID 2LJ7) that was obtained by solution NMR technique (Berman et al., 2000). The antimicrobial peptide Lc-def contains 47 residues, from which eight are cysteines that form four intramolecular disulfide bonds (Fig. 1) and stabilize an antiparallel $\beta$-sheet conformation flanked by an $\alpha$-helical segment (Fig. 2).

The structure was solvated in water boxes with shape of the dodecahedron. The simulation experiments were performed on an IBM X3950 server (Butu and Butu, 2010; 2011b). The program used for these MD simulations was GROMACS software package (Kutzner C. et al., 2007; Pronk et al., 2013), the forces field used was AMBER99SB protein (Hornak et al., 2006). The water model was a modified TIP3P model adapted for these versions of Gromacs. The starting coordinates taken from Protein Data Bank were prepared for Gromacs software as follows: were added $\mathrm{H}$ atoms and for neutralizing the electric charge were added chloride ions.

The resulted systems had been minimized using steepest descent algorithm (SD) for 10000 steps. After this, the systems were heated from $0 \mathrm{~K}$ to $500 \mathrm{~K}$ by rescale velocities for $200 \mathrm{ps}$. After heating, the systems were minimized again using SD algorithm for 10000 steps and gone to equilibration for a $100 \mathrm{ps}$ in NVT and for a 100 ps in NPT. Finally, was started the molecular dynamics simulation which was performed using Periodic Boundary Conditions and number of molecules $\mathrm{N}$, pressure $\mathrm{P}$ and temperature $\mathrm{T}$ were held constant. During the simulation, the length of bindings that involve hydrogen was constrained by LINCS algorithm. The resulting trajectory length for production dynamics had $100 \mathrm{~ns}$ with a step of $2 \mathrm{fs}$. The energy values and the coordinates were saved on every 2 ps. The simulation analysis was done with Gromacs and VMD programme (Hsin et al., 2008).

\section{RESULTS AND DISCUSSION}

For trajectories recorded in simulation experiments were analyzed various structural, dynamic and thermodynamic parameters, including sequence, size, root mean square distances, accessibility surface area, dihedrals, distances between $\mathrm{C} \alpha$ atoms, disulfide bridges and hydrogen bounds.

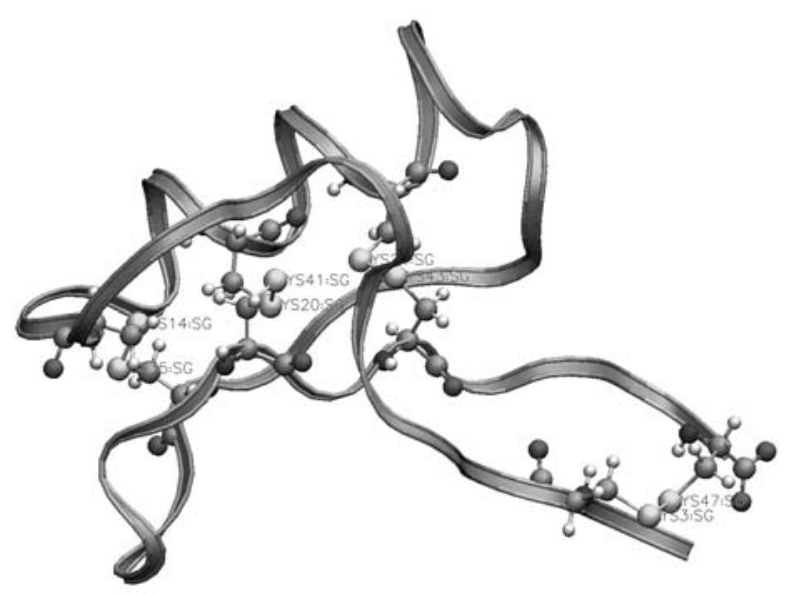

Fig. 1. The 3D structure of the defensin Lc-def: ribon representation of backbone and CPK representation of Cys residues
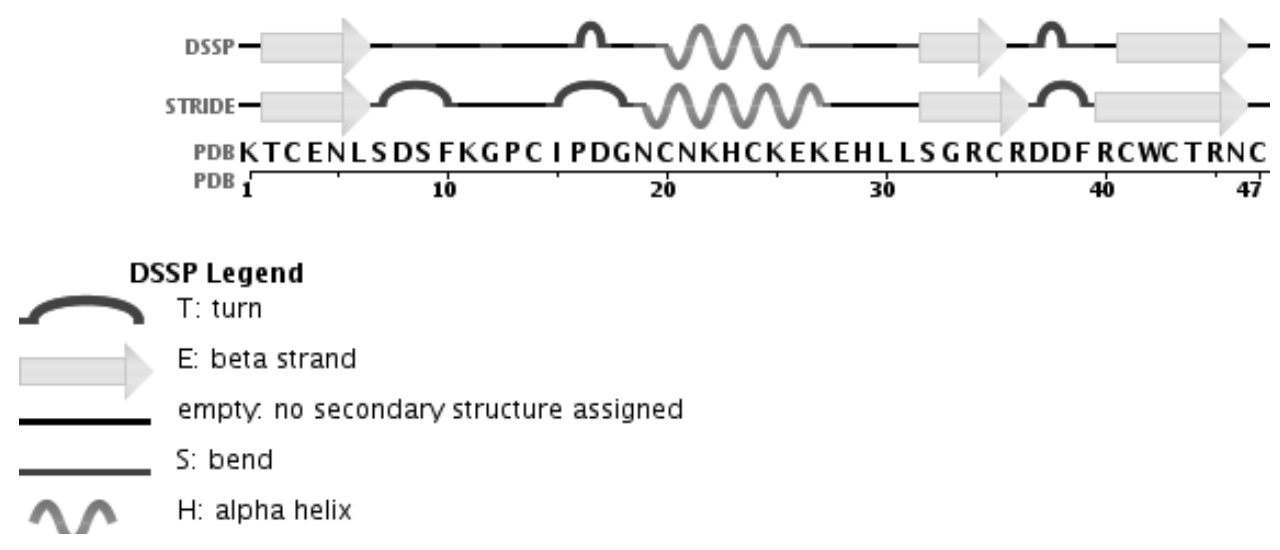

Fig. 2. The detailed SEQRES sequence of the defensin Lc-def 
Root mean square distance over the entire simulation was $5.63 \AA$ for all peptide atoms, 4.76 $\AA$ for main chain atoms and $6.16 \AA$ for side chain atoms (Fig. 3.a). The average values of accessibility surface area for all protein are hydrophobic ASA $11.37 \mathrm{~nm}^{2}$, hydrophilic ASA $14.29 \mathrm{~nm}^{2}$ and total ASA $26.24 \mathrm{~nm}^{2}$ (Fig. 3.b).

The analysis of root mean square distances for each residue after completing the simulation revealed four types of dynamic behavior, namely: unstable residue (Fig. 4.a); residues which present two stable states between which they fluctuate with high frequency (Fig. 4.b); residues which presents two stable states between which they fluctuate with low frequency (Fig. 4.C) and stable residues (Fig. 4.d). Comparing the residues behavior with the secondary structure assigned, it was found that the unstable residues are positioned at the entrance and exit of the beta strand structures. The residues that have two stable states between which they fluctuate with high frequency are positioned inside the beta strand structures. The residues that have two stable states between which they fluctuate with low frequency are part of the turn or beta strand structures. The stable residues belong to the alpha helix structure. The four types of behavior are illustrated in Figure 4.

The secondary structure changes that occur in the dynamics can be observed by drawing the Ramachandran plots for each residue. Thus, in the studied structure is observed that the dihedrals which change take values belonging to two distinct areas and feature two types of behaviors: a) dihedrals which shows a higher share in one area, namely, in the characteristic area of the beta strand structure values (Fig. 5. $a$ and $b$ ) dihedrals which shows a relatively equal share for the two areas (Fig. 5.b).

The behavior in dynamic of the secondary structure of the antimicrobial peptide can be observed in 3D Ramachandran plot (Fig. 6.a). The values recorded by the dihedrals during the simulation are directly related to the types of secondary structures and their weight (Fig. 6).

The investigated parameters are influencing the biological activity and function of the AMPs-P. The conformational changes were characterized for the entire peptide structure and for the local structure, also.

\section{CONCLUSION}

For the trajectories resulted from the molecular dynamics simulation the dynamic and thermodynamic parameters were analyzed. The results lead to the conclusion that in silico analysis of AMPs-P can identify optimal conformation of those biomolecules. In addition, this analysis can be used in design of the mutants with optimal conformations for fulfilling certain biological functions in specified conditions (temperature, pressure, $\mathrm{pH}$, etc) or to develop new biotechnological applications of the AMPs-P in medicine and plant protection.

Acknowledgments. This work was supported by the UEFISCDI, Program 4 - Partnerships in Priority S\&T Areas/ 2nd National Plan for Research, Development \& Innovation, research contract PT-PCCA 106/2012.

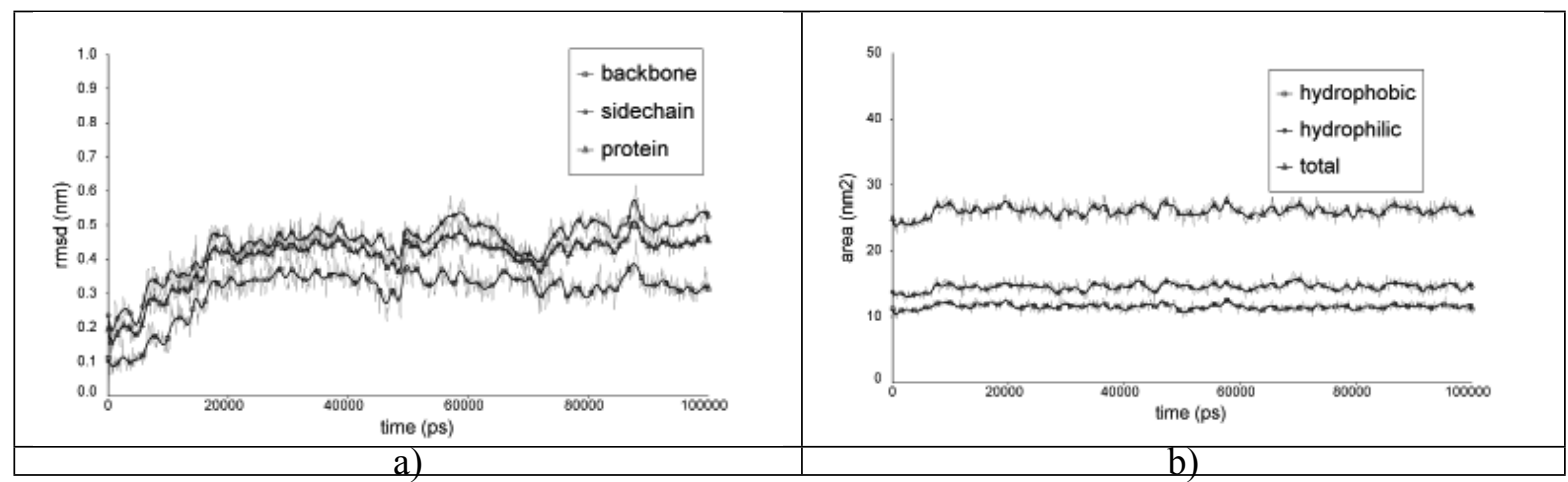

Fig. 3. Evolution in dynamics of: a) root mean square distances for backbone, sidechain and protein; b) hydrophobic, hydrophilic and total accessibility surface areas. 


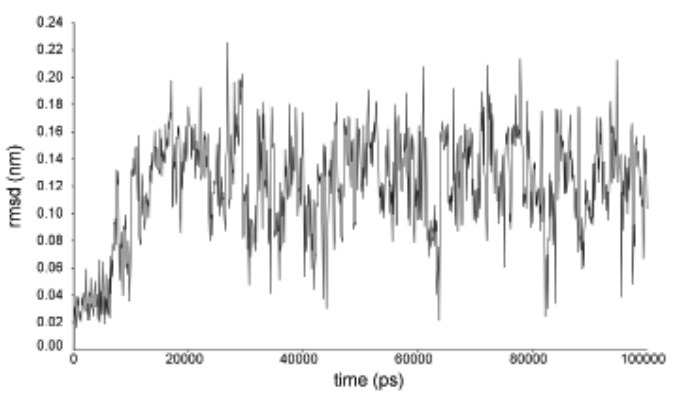

SER 9

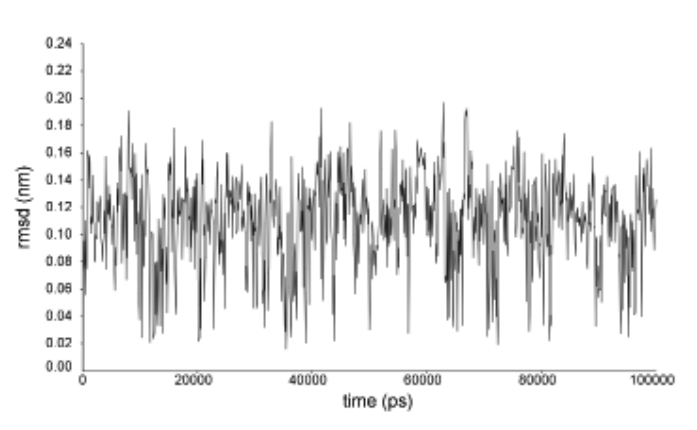

LEU 31

a) unstable residues

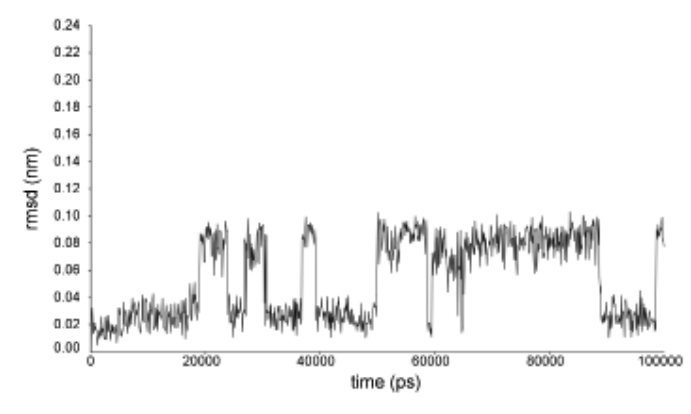

CYS 3

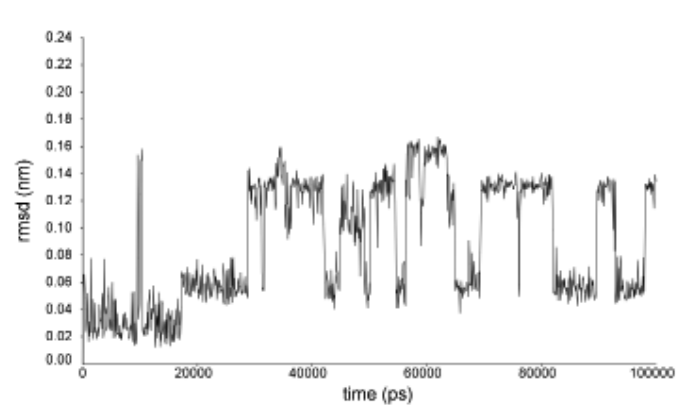

GLY 33

b) residues which has two stable states between fluctuates with high frequency

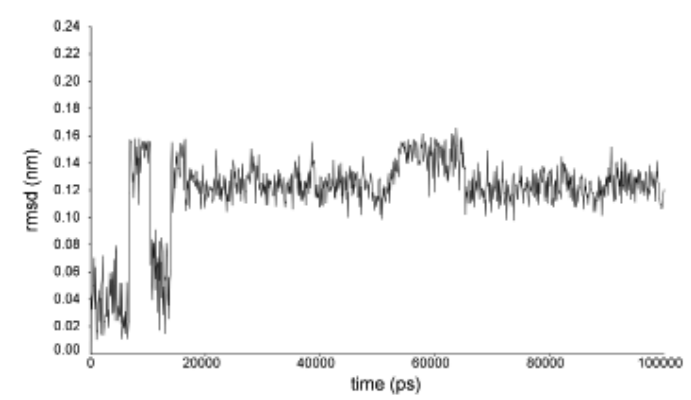

SER 32

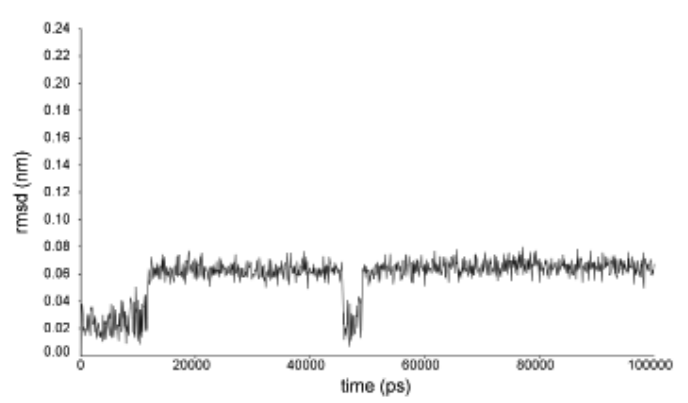

LEU 6

c) residues which has two stable states between fluctuates with low frequency

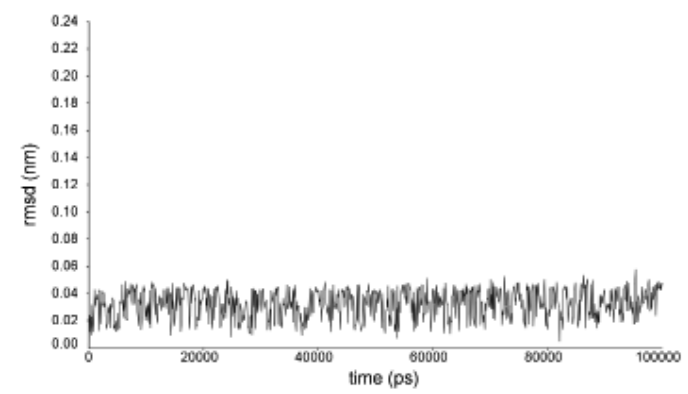

HYS 29

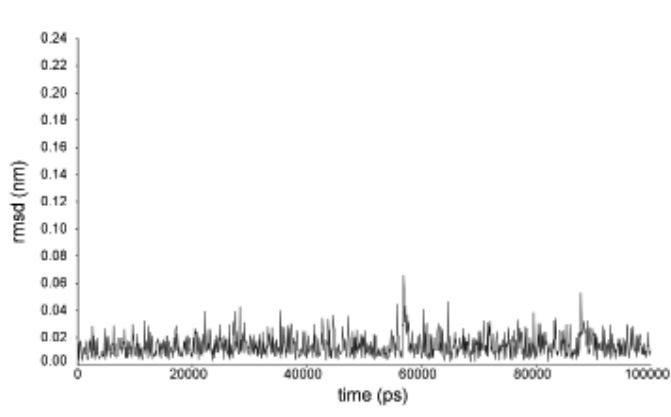

ASP 38

d) stable residues

Fig. 4. Evolution of RMSD for residues. 

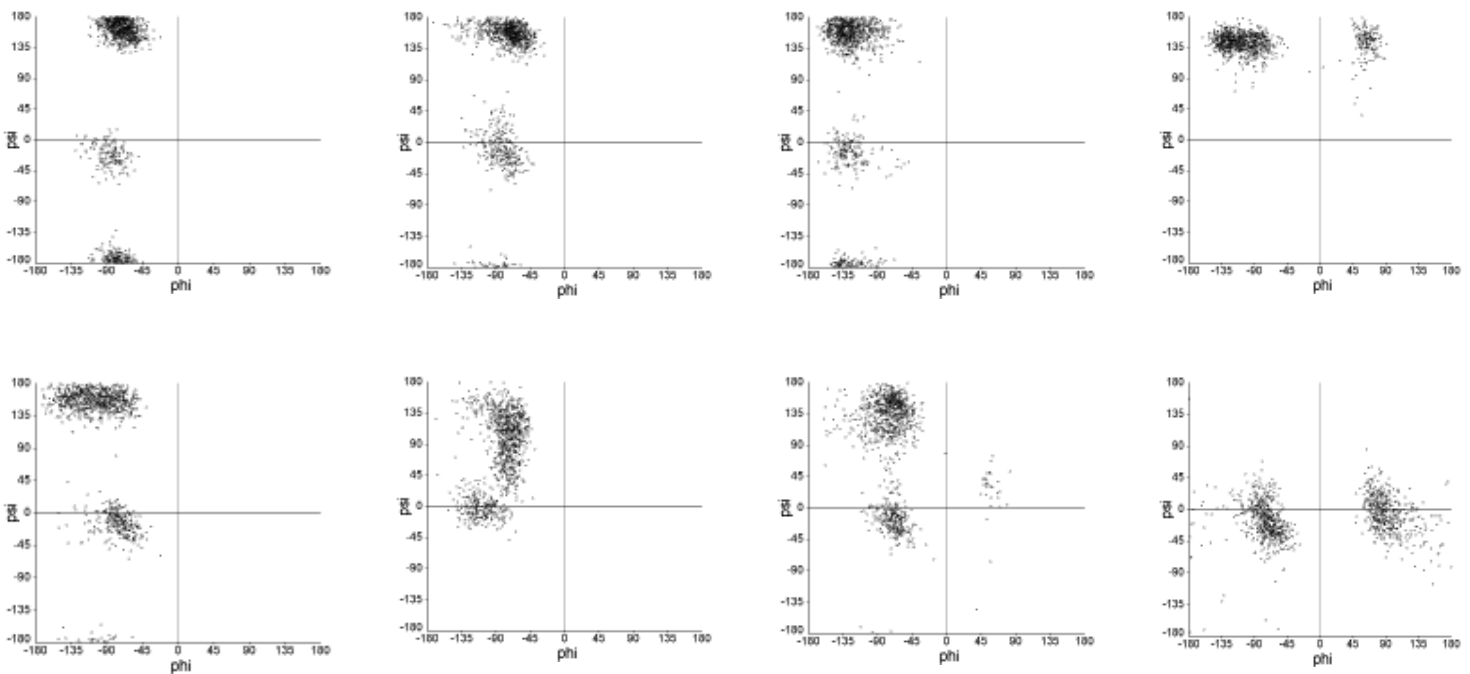

Fig. 5. The Ramachandran plot for residues.

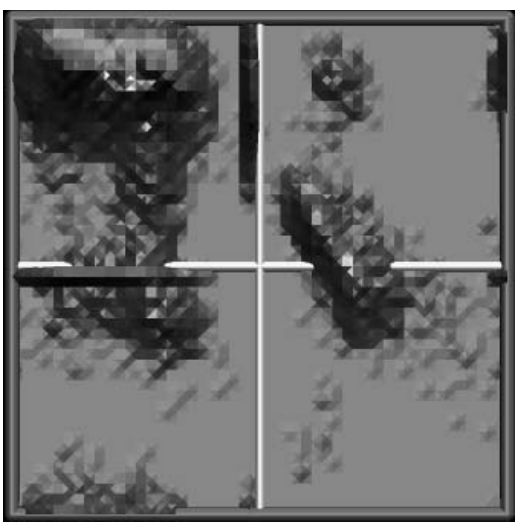

a)

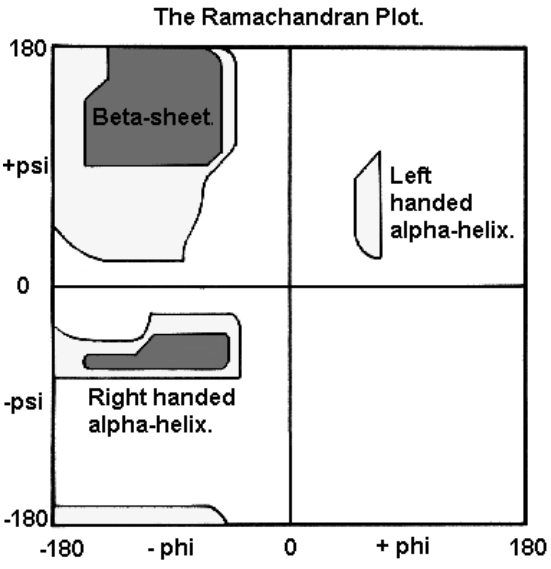

b)

Fig. 6. The Ramachandran plot: a) 3D distribution of dihedrals of 2LJ7

for entire simulated trajectory; b) distribution of dihedrals with respect to the secondary structure.

\section{REFERENCES}

1. Berman HM, Westbrook J, Feng Z, Gilliland G, Bhat TN, Weissig H, Shindyalov IN and Bourne PE (2000). The Protein Data Bank, Nucleic Acids Research, 28: 235-242.

2. Butu M and Butu A (2010). Parallel molecular dynamics simulation for protein sequences on PC-cluster and server Journal of Optoelectronics and Advanced Materials, 12(11): 2296-2300.

3. Butu M and Butu A (2011a). Antimicrobial peptidesnatural antibiotics, Romanian Biotechnological Letter, 16(3): 6135-6144.

4. Butu M and Butu A (2011b). Molecular dynamics simulation of the Human Alpha-Defensin 5, Digest Journal of Nanomaterials and Biostructures, 6(3): 907-914.

5. Hornak V, Abel R, Okur A, Strockbine B, Roitberg A and Simmerling C (2006). Comparison of multiple Amber force fields and development of improved protein backbone parameters. Proteins, 65: 712-725.
6. Hsin J, Arkhipov A, Yin Y, Stone JE and Schulten K (2008). Using VMD: an introductory tutorial, Current Protocol in Bioinformatics, Chapter: unit 5.7.

7. Kutzner C. van der Spoel D, Fechner M, Lindahl E, Schmitt $\mathrm{U}$, de Groot B and Grubmüler H (2007). Speeding up parallel GROMACS on high-latency networks. J. Comp. Chem, 28 (12): 2075-2084.

8. de Oliveira Carvalho A and Moreira Gomes V (2009). Plant defensins -Prospects for the biological functions and biotechnological properties, Peptides, 30: 1007-1020.

9. Pronk S, Páll S, Schulz R, Larsson P, Bjelkmar P, Apostolov R, Shirts MR, Smith JC, Kasson PM, van der Spoel D, Hess B and Lindahl E (2013). GROMACS 4.5: A high-throughput and highly parallel open source molecular simulation toolkit. Bioinformatics 29: 845-854.

10. van der Weerden NL and Anderson M (2014). Plant defensins: Common fold, multiple functions, Fungal Biology Reviews, 26: 121-131. 